\title{
Analysis of spine loads in dentistry-impact of an altered sitting position of the dentist
}

\author{
Max Wunderlich ${ }^{1}$, Thomas Eger ${ }^{2}$, Thomas Rüther $^{1}$, Andreas Meyer-Falcke ${ }^{3}$, Dieter Leyk ${ }^{1,4}$ \\ ${ }^{1}$ German Sport University Cologne, Department of Physiology and Anatomy, Cologne, Germany; \\ ${ }^{2}$ Department of Periodontology, German Armed Forces Central Hospital, Koblenz, Germany; \\ ${ }^{3}$ Health Care Campus North Rhine-Westphalia, Universitätsstraße 136, Bochum, Germany; \\ ${ }^{4}$ Department IV - Military Ergonomics and Exercise Physiology, German Armed Forces, Koblenz, Germany. \\ Email: M.Wunderlich@dshs-koeln.de; Ruether@dshs-kolen.de; Leyk@dshs-koeln.de; ThomasEger@,bundeswehr.org; \\ andreas.meyer-falcke@gc.nrw.de; DieterLeyk@bundeswehr.org
}

Received 25 March 2010; revised 6 April 2010; accepted 8 April 2010.

\section{ABSTRACT}

Neck and low back pain causes highest disability rates in industrialized countries. Apart from bluecollar occupations dentists and dental care personnel are also strongly affected by work related back pain. However, due to missing analysing systems spine exposure could not be adequately estimated for sitting and non load bearing tasks. Therefore, a new biomechanical measurement system was developed to analyze spine and trunk kinematics. To explore the potential kinematics impact on the spine two days of periodontology care have been examined. Moreover, to asses the influence of an altered sitting position data was collected in 9 (day 1) and 12 (day 2) o'clock position. Data were collected and analyzed by the new 3D-SpineMoveGuard. The systems analyze postures and movements of spine and trunk by ultrasound and inclinometer. Two days of routine nonsurgical periodontal treatment were recorded by a frequency of $10 \mathrm{~Hz}$. Moderate awkward postures could be assessed for $90 \%$ of the treatment. Up to $85 \%$ of the working days were spent in isometric position. Movements were rare and of small amplitude. There were no relevant differences between 9 and 12 o'clock position. The frequently reported back pain in dentists is not only related to severe body postures rather than to the high amount of isometric spine loads. Moreover, an altered sitting position did not reduce this physiological stress. Therefore, dentist specific interventions concepts should pay more attention to physical training during and off work. Ergonomic investments should be carefully evaluated before implemented.

Keywords: Occupational Medicine; Periodontal Treat- ment; Back Pain, Ergonomics; 3D-SpineMoveGuard

\section{INTRODUCTION}

About $80 \%$ of the adult population of industrialized countries are affected by at least some episode of back pain $[1,2]$. The highest prevalence rates can be estimated for employees in the "mid-ages". Moreover, the major economic burden results from the high disability rates and the persistent recurrence of the preliminary cervical or lumbar pain $[3,4]$. As a large part of these disabilities are caused by specific job demands preventive strategies have to focus on the reduction of occupational stressors to avoid chronic episodes of back pain.

The most common causes for occupation related back pain are biomechanics impacts. Beside the negative effects of load bearing tasks, there is evidence from epidemiological studies that awkward trunk postures (bending, twisting, isometrics, repetitive movements) are the most reasonable cause for work related back pain $[5,6]$. Furthermore, numerous of psycho-social co-factors could be estimated to intensify the pain episode or to be involved in the recurrence of the unspecific pain.

Back pain in dentistry

Regarding the existing tools to assess spine loads at the workplace it appears that the tasks which dental workers routinely perform are at low risk for ergonomically related disorders [7]. This rating is founded because of the probably good environmental conditions (no cold or wet climate), excellent individual job motivation and social background, no load bearing tasks, no extreme body posture etc. However, low back and neck pain is a severe and common occupation related injury in dentistry. Survey data revealed a one year prevalence of back and neck pain of about 70\%-90\% [8-10]. Moreover, Wunderlich et al. [10] could estimate that about $80 \%$ of 
the affected German military dentist population occupy medical help to deal with the pain. These data indicate that back pain is a major health problem for dental practitioners and methods are needed objectifying the causes and to identify appropriate intervention strategies [11]. With regard to ergonomic improvements studies indicated that a specific treatment position ("9 o'clock" vs. "12 o'clock") or a specific dentist's stool directly affects the impact on the spine and the dentist's health $[8,12]$. However, up to date potential risk factors for musculoskeletal disorders (MSD) in dentistry were only assessed due to questionnaires and qualitative reports of observation studies [13]. As long as only a rough and almost invalid analysis could be done it seems unlikely to objectify the real biomechanics impact on the spine and to justify useful preventive or ergonomic interventions.

The aim of the present explorative study was to assess information in detail on trunk posture and movements by a newly developed ultrasound based mobile device: 3D-SpineMoveGuard (3-D SMG). Beside the objective information on the working posture and spine kinematics two different treatment positions ("9 o'clock" vs. " 12 o'clock") where compared to give insight in a potentially less stressful working posture.

\section{MATERIALS AND METHODS}

The present study was proven and positively evaluated by the ethics committee of the German Sport University Cologne. Every subject voluntarily participated in the study and gave their written informed consent.

\subsection{The 3-Dimensional SpineMoveGuard (3D-SMG)}

Movements and postures of the trunk and spine were captured with the $3 \mathrm{D}-\mathrm{SMG}$ system. The $3 \mathrm{D}-\mathrm{SMG}$ is a further developed ultrasound based mobile measurement device to assess three dimensional posture and movement profiles of spine and trunk in real-time. Moreover, to standardise data analysis and to reveal new kinematic parameters the analysing software "JSpinal" was developed.

The basic component of the 3D-SMG is the ultrasound device "sonoSens ${ }^{\circledR}$ Monitor" (Friendly Sensors AG, Jena, Germany). Details of the device are described elsewhere [14,15]. In brief: Four pairs of ultrasound transmitters and receivers (diameter $20 \mathrm{~mm}$, height 4 $\mathrm{mm}$ ) were fixated on the skin (Figure 1) with adhesive tape. For each channel the skin distance between transmitter and receiver is determined at a sampling rate of 10 $\mathrm{Hz}$. The transmitters and receivers are cable connected to a small data logger. Variations in inter transmitter skin distance (shorter or longer running times of the ultra

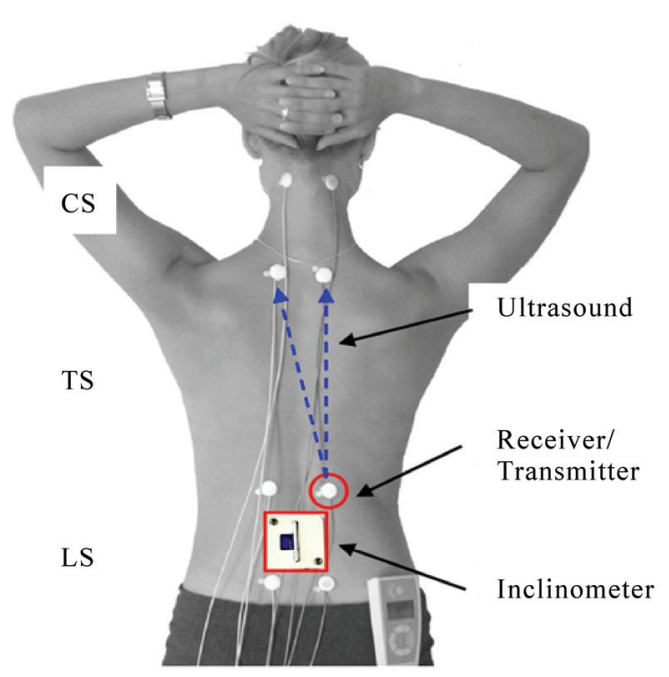

Figure 1. Hardware application of the 3D-SpineMoveGuard. Ultrasound transmitters/receivers and the sagittal inclinometer have their defined positions at the cervical (CS), thoracic (TS) and lumbar spine (LS).

sound signal) represent a change in spine posture within the associated segment of the spine (Figure 1). The data points of the 12 channel system represent a 3D model (sagittal-, frontal- and horizontal plane) of the external spine curvature and can be stored for up to 10 hours.

In addition to the changes in spine curvature knowledge of the independent occurrence of trunk inclination is important. Therefore, a one dimensional capacitive, dielectric liquid-based inclinometer (NA4-70, Seika Mikrosystemtechnik GmbH, Kempten, Germany) was taped to the skin with the top edge at the middle of the spinous process of the $2^{\text {nd }}$ lumbar vertebra (Figure 1). The inclinometer is cable-connected to a data logger. The range of measurement was adjusted between $+110^{\circ}$ and $-30^{\circ}$ (frontal/dorsal). Data was captured at a sampling rate of $10 \mathrm{~Hz}$.

\subsection{The Analyzing Software "JSpinal"}

The newly developed analyzing software JSpinal enables the valid data management and the detection of movements and postures. JSpinal analyses the segmental data to

1) adjust the row data with the occupation specific reference position (segmental spine length in upright sitting position).

2) calculate relative (\%) length indices to separately detect sagittal (SLI), frontal (FLI) and horizontal (HLI) movements or postures. The relative (\%) spine curvature enables the comparison of individuals with different spine proportion. 
3) automatically detect the direction of deviations from the reference position (neutral zero): SLI forward (flexion)/backward (extension), FLI lateral flexion right/left and HLI torsion right/left

4) analyze the frequency distribution of spine and trunk posture.

5) analyze the frequency and the amount of combined spine postures: detecting the deviation from neutral zero in at least two body planes independent of the direction (e.g. flexion + torsion or torsion + lateral flexion etc.).

6) analyze the kinematic characteristic of the data: duration, frequency and magnitude of movements as well as duration, frequency and spine/trunk position of isometric postures. Both, dynamic and isometric parameters were analysed within every single spine segment and body plane

7) rate the variability of the occupational demands data can be chronologically analysed. Therefore the time of the analysis will be divided in to 10 segments (percentiles of the time) though the intensity/frequency of spinal stressors can be compared between the segments.

\subsection{Subject and Examination Procedure}

The explorative assessment of spine loads in dentistry was conducted by a single case study. Data collection was done on the same days of two consecutive weeks. At the first day data were assessed in the 9 o'clock position; at the second date in the 12 o'clock position. To keep the medical treatments comparable only 20 patients for periodontol maintenance were recruited for the two examination days. All patientes were treated with two different hand scalers, curettes, ultrasonic devices, rotating and polishing instruments. Each treatment session lasted 45 minutes.

After the instrumentation of the measurement devices maximal voluntary range of movements were assessed within the three body planes in standardised positions [14]. Furthermore, to identify the reference working position (neutral zero) the dentist set down on his working stool with his hands relaxed on his thighs and an upright trunk position with the head held according to the Frankfurt horizontal. Each of the positions was kept for about 30 seconds. Shortly thereafter the routinely treatment of the patients began in the usual and necessary duration and velocity.

\subsection{Statistics}

Data analyses were reduced to the time of treatment procedure. Periods between the treatments and breaks were excluded to reduce the bias due to individual behaviouristic. Because of the explorative character of the study only descriptive data analyses were done by SPSS $C$
17.0. Data is shown as frequency and percentile $\left(5^{\text {th }}, 25^{\text {th }}\right.$, median, $75^{\text {th }}$, and $\left.95^{\text {th }}\right)$ distributions.

\section{RESULTS}

The maximal voluntary range of motion is shown in Table 1 . The magnitudes can be used to compare the deviations from neutral zero (upright sitting) during the non surgical periodontal treatment.

Figure 2 indicates the percentile values of spine and trunk positions throughout the whole working day. The different profiles of the 9 and 12 o'clock working position indicate the necessary adoption of the posture by the dentist. In detail:

Cervical spine (CS): sagittal deviations from neutral zero are very small. The spectrum of lateral flexion and torsion is about 3 times broader in both 9 and 12 o'clock positions. In 9 o'clock position FLI and HLI values are almost normal distributed around zero. During the 12 o' clock manoeuvre the CS is in $75 \%$ of the treatment time in a lateral left position and turned to the right.

Thoracic spine (TS): the range of movement is very small. The TS were held in the almost identical position in both 9 and 12 o'clock position. During the 12 o'clock treatment FLI and HLI data ranges in a contra lateral relation to the values of CS.

Lumbar spine (LS): the range of motion is moderate and between the spectrum of CS and TS. In 9 o'clock SLI and FLI indicate an almost upright sitting position. HLI values show a continuous left rotation toward the patient. During 12 o'clock treatment SLI is in a flexed position during $75 \%$ of the treatments (the head of the patient was in front of the abdomen of the dentist). Moreover, LS is laterally flexed to the right (FLI) and rotated to the left during the whole working day.

Trunk inclination (TI): the range of trunk motion in 9 o'clock indicating a broader spectrum of trunk positions and is almost normal distributed. In 12 o' clock TI is negative in $75 \%$ of the treatments, indicating the tilt of the pelvis.

Table 1. Maximum voluntary range of motion for each part of the spine and within the three body planes.

\begin{tabular}{|c|c|c|c|}
\hline \multirow[t]{2}{*}{ Body planes } & \multicolumn{3}{|c|}{$\begin{array}{c}\text { Maximum voluntary range of } \\
\text { motion } \\
\text { upright sitting }=0 \%\end{array}$} \\
\hline & CS & TS & LS \\
\hline Sagittal plane (Extension/Flexion) & $-25 \% / 24 \%$ & $-8 \% / 20 \%$ & $-10 \% / 22 \%$ \\
\hline Frontal plane (left/right) & $-18 \% / 21 \%$ & $-16 \% / 17 \%$ & $-15 \% / 16 \%$ \\
\hline Horizontal plane (left/right) & $-20 \% / 21 \%$ & $-8 \% / 10 \%$ & $-8 \% / 12 \%$ \\
\hline
\end{tabular}

$\mathrm{CS}=$ cervical spine $; \mathrm{TS}=$ thoracic spine $; \mathrm{LS}=$ lumbar spine 


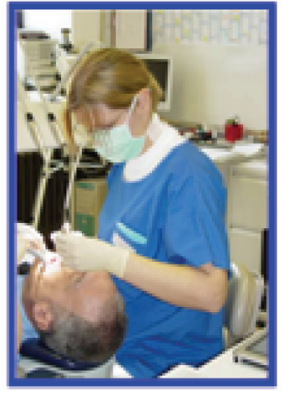

9 o'clock position

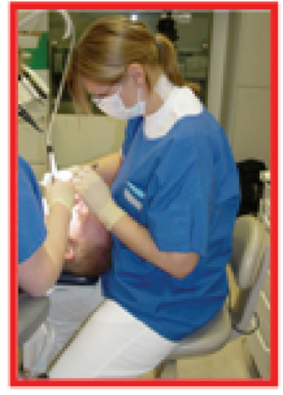

12 o'clock position

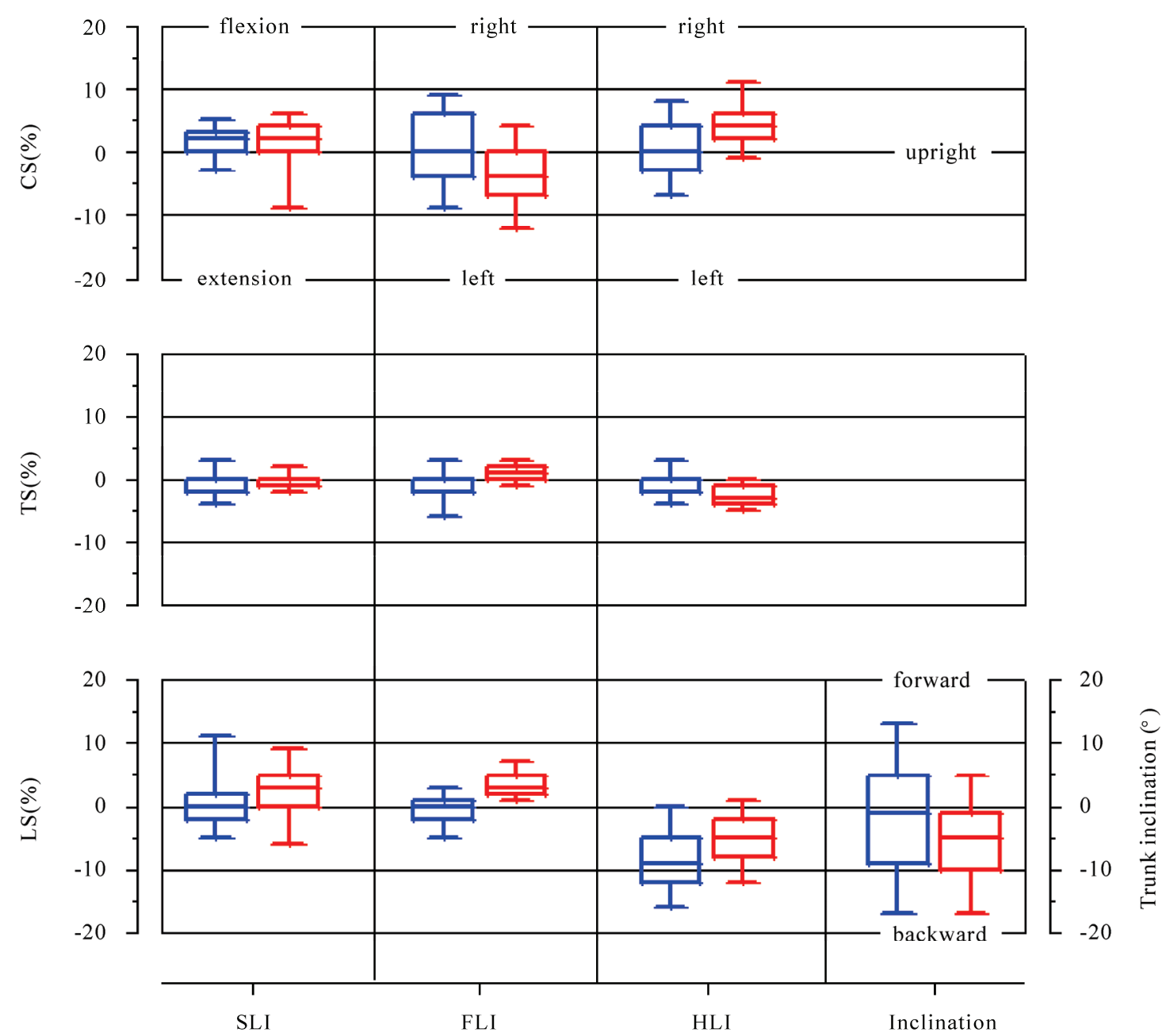

Figure 2. Distributions of segmental spine positions of the dentist in 9 o'clock (blue/left symbol) and 12 o'clock treatment positions. Deviations from upright sitting " 0 " can be determined for sagittal (SLI), frontal (FLI) and horizontal (HLI) spine and trunk (sagittal inclination) postures. Box-Plots are showing $5^{\text {th }}, 25^{\text {th }}, 50^{\text {th }}, 75^{\text {th }}$, and $95^{\text {th }}$ percentile for cervical (CS), thoracic (TS) and lumbar (LS) spine.

Apart from the postures within one body plane the analyses further revealed the amount of "combined postures". Figure 3 shows the frequency of combined spine postures during periodontology care in 9 and 12 o'clock position. The postures are grouped from "small" $(0-2 \%)$, "medium" (> 2-6\%), "large" (> 6-10\%) to "extreme" $(>$ 

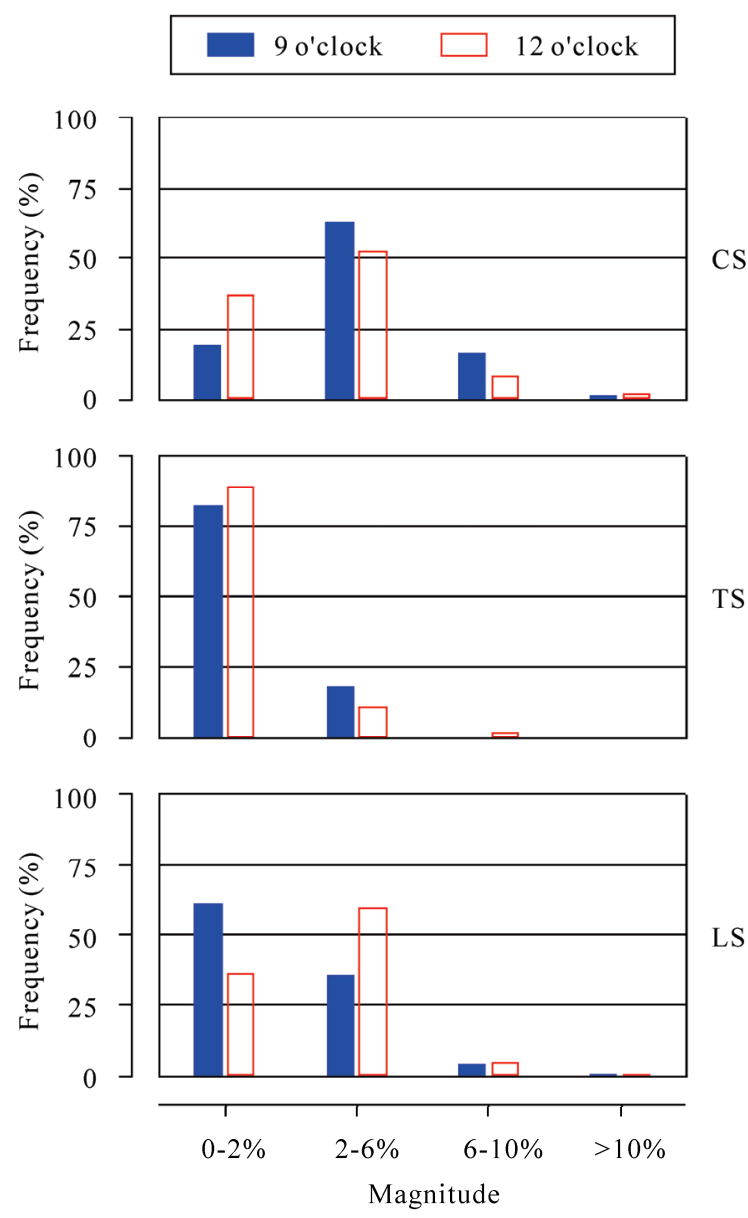

Figure 3. Frequency of multi-directional combined postures of the spine. Values are shown depending on magnitude (deviation from upright sitting) of the combined postures for cervical (CS), thoracic (TS) and lumbar (LS) spine.

$10 \%$ ). During $90 \%$ of the treatment time the combined postures ranged between "small" and "medium" with clear differences between the segments of the spine. Position related ( 9 vs. 12 o'clock) varieties could only be estimated for the LS, where about $2 / 3$ of the treatment time in 12 o'clock position where spend in a "medium" combined posture.

The further analysis of the kinematic work demand revealed that in more than $75 \%$ of the treatment time no spine movements occur. Small or medium movements of the trunk could be estimated in about $25 \%$ of the work time (Table 2). In addition, during $75 \%$ to $85 \%$ of the periodontology care the dentist worked in an isometric posture (Figure 4). As could be drawn from Figure 3 there exit some differences in static work posture between the segments of the spine but none between treatment positions of the dentist (9 vs. 12 o'clock). Moreover, Figure 5 shows the durations (s) of isometric

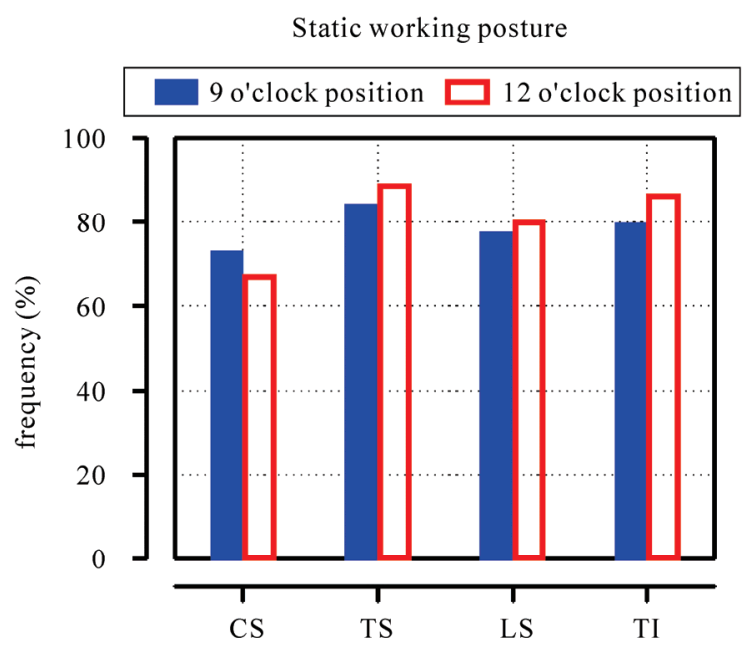

Figure 4. Frequency of static work posture during periodontologic care. Values are shown for cervical (CS), thoracic (TS) and lumbar (LS) spine as well as trunk inclination (TI).

Duration of isometric work postures
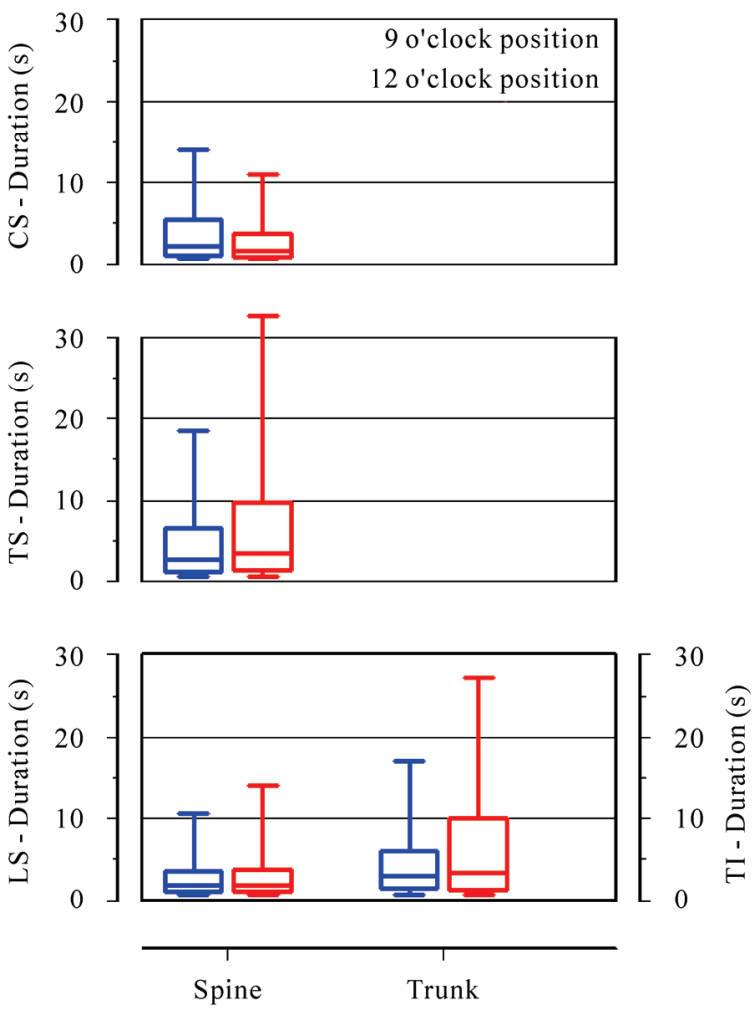

Figure 5. Duration of isometric work postures within the cervical (CS), thoracic (TS) and lumbar (LS) spine as well as for trunk inclination (TI). Box-Plots are showing $5^{\text {th }}, 25^{\text {th }}, 50^{\text {th }}, 75^{\text {th }}$, and $95^{\text {th }}$ percentile. 
Table 2. Distribution of movement amplitudes of the cervical (CS), thoracic (TS) and lumbar (LS) spine. The amplitudes represent relative (\%) differences in segmental spine length per movement. Amplitudes of the trunk are expressed as degree $\left({ }^{\circ}\right)$ changes per movement.

\begin{tabular}{|c|c|c|c|c|c|c|c|c|}
\hline \multirow{3}{*}{ Percentile } & \multicolumn{8}{|c|}{ Distribution of movement amplitudes } \\
\hline & \multicolumn{2}{|c|}{ Amplitude (\%) CS } & \multicolumn{2}{|c|}{ Amplitude (\%) TS } & \multicolumn{2}{|c|}{ Amplitude (\%) LS } & \multicolumn{2}{|c|}{ Amplitude $\left({ }^{\circ}\right)$ Inclination } \\
\hline & 9 o'clock & 12 o'clock & 9 o'clock & 12 o'clock & 9 o'clock & 12 o'clock & 9 o'clock & 12 o'clock \\
\hline 5. &, 00 &, 00 &, 00 & 00 &, 00 &, 00 &, 00 &, 00 \\
\hline 25. &, 00 &, 00 &, 07 & 07 & 02 &, 03 &, 00 &, 00 \\
\hline 50. &, 22 &, 20 &, 16 &, 16 &, 14 &, 15 & 3,00 & 3,00 \\
\hline 75. &, 44 &, 44 &, 54 & 48 & 40 &, 34 & 5,00 & 5,00 \\
\hline 95. & 3,07 & 3,54 & 2,28 & 1,74 & 2,51 & 2,51 & 14,00 & 11,00 \\
\hline \multicolumn{4}{|c|}{ Rating of spine movements } & \multicolumn{5}{|c|}{ Rating of trunk inclination movements } \\
\hline \multicolumn{4}{|c|}{$0-<2 \%=$ "no movement" } & \multicolumn{5}{|c|}{$0^{\circ}-<3^{\circ}=$ "no movement" } \\
\hline \multicolumn{4}{|c|}{$2-6 \%=$ small to medium amplitude } & \multicolumn{5}{|c|}{$3-20^{\circ}=$ small to medium amplitude } \\
\hline \multicolumn{9}{|c|}{$>6 \%=$ large amplitude $\quad>20^{\circ}=$ large amplitude } \\
\hline
\end{tabular}

work postures. The median values could be estimated by 3 to 4 seconds for both spine and trunk. However, interruptions where even shorter (median duration of movements $\approx 1 \mathrm{~s}$ ) and as shown in Table 2 of small amplitudes.

\section{DISCUSSION}

During periodontology care spine and trunk of the dentist where held within a small range of movement. The deviation from neutral zero was very small in TS and higher in CS and LS. The frequently postulated assumption that dentists work in a sewer bended and twisted posture $[8,11]$ could not be confirmed. During $90 \%$ of the non-surgical treatments the combined postures ranged between "small" and "medium" deviation from neutral zero. It turned out that the preliminary posture specific risk factor could be the continuous phases of isometric spine and trunk position. Moreover, the changes of work postures were done very slowly and by small movements. The revealed kinematics and postures of spine and trunk differed only slightly between the most popular treatment positions $(9$ o'clock vs. 12 o'clock) of the dentist.

As it has been frequently reported more than $90 \%$ of the back pain is unspecific: No structural damage or injury can be detected to explain the cause of the aches [16]. Hence, the pain seems to be triggered by the active and/or passive structures of the back and spine. In this context it seems important to remember that the muscles are much more than a contractile organ. The muscles are rather a large sensory part of the body which registers a broad spectrum of chemical and mechanical changes $[17,18]$.

With regard to the new quantitative findings of the task analysis the static none neutral work postures can lead to reduced muscle circulation. As a result metabolites accumulate within the extracellular space. Beside these perfusion and metabolic related volumes and concentration shifts mechanical pressure to the tissue could also lead to acute or delayed exposure induced pain $[17,19]$. Chemical or mechanical changes will be registered by free interstitial nerve endings (chemo and mechano receptors) of slow afferent nerve fibres (group III and IV). This will not only lead to an increase in heart circulation and breathing but can also reveal reflectively pain phenomena and changes in muscle tension $[17,19]$. In addition to these acute reactions prolonged static none neutral posture can also cause structural damage of the spine. Additionally, none neutral work posture of the dentist leads to a continuous eccentric exposure of the intervertebral disc. Static and eccentric pressures to the disc can lead to deficits in locale nutrition, metabolic imbalance and can cause cumulative trauma within the structure of the disc and the vertebral end plates [20].

Former studies concluded that changes in treatment positions of the dentist and/or ergonomic sitting stools and/or arrangements of the medical practice will lead to significant reductions in spine exposure $[8,12]$. However, the detailed data of the explorative study did not reveal substantial differences of exposure relevant body postures due to an altered treatment position of the dentist. 
Moreover, the data suggests that dental care is per se strongly associated with a certain, uncomfortable work posture and spinal loads. Intervention concepts should consider both ergonomics and individual alterations. To be more precisely, specific physical intervention concepts have to be adapted for dental care personnel to cope with the physical stress of work postures. The activity programs have also to be adapted to the daily routine of the personnel to interrupt and reduce the cumulative isometric load to the spine $[21,22]$.

The results of the present explorative study are of course not representative for body postures during dental care. Moreover, due to limited methodical capabilities of former studies the parameters are not directly comparable with the literature. But to the best of our knowledge there is no comparable detailed data of kinematics parameter of trunk and spine during dental care. However, the data documents exemplarily the task specific work load characteristic, enables the objective evaluation of interventions (sitting position) and leads to occupation relevant intervention concepts.

\section{CONCLUSIONS}

The frequently reported back pain in dentist is not only related to severe body postures rather than to the high amount of isometric spine loads. Moreover, with regard to an altered sitting position of the dentist only slightly exposure relevant differences could be estimated. Therefore, intervention concepts for dental care personnel should pay more attention to individual physical training during and off work.

\section{ACKNOWLEDGEMENTS}

The analysis was funded by the Medical Service of German Armed Forces (M/SAB1/4/A009) and supported by the Department of Periodontology of the German Armed Forces Central Hospital Koblenz.

\section{CONFLICT OF INTEREST}

The authors declare that they have no conflict of interest.

\section{REFERENCES}

[1] Schneider, S., Schmitt, H., Zoller, S. and Schiltenwolf, M. (2005) Workplace stress, lifestyle and social factors as correlates of back pain: A representative study of the German working population. International Archives of Occupational and Environmental Health, 78(4), 253-269.

[2] Schneider, S. and Schiltenwolf, M. (2006) Occupations associated with a high risk of self-reported back pain: Representative outcomes of a back pain prevalence study in the Federal Republic of Germany. European Spine Journal, 15(6), 821-833.

[3] Kumar, S. (2001) Theories of musculoskeletal injury causation. Ergonomics, 44(1), 17-47.

[4] Juniper, M., Le, T.K. and Mladsi, D. (2009) The epide- miology, economic burden, and pharmacological treatment of chronic low back pain in France, Germany, Italy, Spain and the UK: A literature-based review. Expert Opinion on Pharmacotherapy, 10(16), 2581-2592.

[5] Harkness, E.F., Macfarlane, G.J., Nahit, E.S., Silman, A.J. and McBeth, J. (2003) Risk factors for new onset low back pain amongst cohorts of newly employed workers. Rheumatology, 42(8), 959-968.

[6] Hoogendoorn, W.E., Bongers, P.M., Vet, H.C.W., de Douwes, M., Koes, B.W., Miedema, M.C. Ariëns, G.A. and Bouter, L.M. (2000) Flexion and rotation of the trunk and lifting at work are risk factors for low back pain: Results of a prospective cohort study. Spine, 25(23), 3087-3092.

[7] Guay, A.H. (1998) Commentry: Ergonomically related disorders in dental practice. Journal of the American Dental Association, 129(2), 184-186.

[8] Thornton, L.J., Barr, A.E., Stuart-Buttle, C., Gaughan, J.P., Wilson, E.R., Jackson, A.D., Wyszynski, C.T. and Smarkola, C. (2008) Perceived musculoskeletal symptoms among dental students in the clinic work environment. Ergonomics, 51(4), 573-586.

[9] Wassan, A.L., Almas, K.A. and Shethri, S.E. (2001) Rückenund Nackenbeschwerden bei Zahnärzten und zahnmedizinischem Personal. Journal of Contemporary Dental Practice, 2(3), 17-30.

[10] Wunderlich, M., Rüther, T., Eßfeld, D., Rohde, U., Eger, T., Leyk, D. (2009) Rückenschmerzen und Wirbelsäulenbelastungen - Befragung des zahnmedizinischen Personals in der Bundeswehr. Wehrmed Mschr, 53(1), 230-234.

[11] Leggat, P.A., Kedjarune, U. and Smith, D.R. (2007) Occupational health problems in modern dentistry: A review. Industrial Health, 45(5), 611-621.

[12] Morita Europe GmbH, J. (2008) Dr. Beach ergonomic concept. Warum sich intuitive Arbeitswege lohnen und Rückenschmerzen nicht sein müssen. $D D Z$, 117(4), 188-189.

[13] Marklin, R.W. and Cherney, K. (2005) Working postures of dentists and dental hygienists. CDA Journal, 33(2), 133-136.

[14] Baum, K., Hoy, S. and Essfeld, D. (1997) Continuous monitoring of spine geometry: A new approach to study back pain in space. International Journal of Sports Medicine, 18(Suppl 4), 331-333.

[15] Friedrich M (2002) Measuring lumbar sagittal posture in sewgae workers using an ultrasonic device. Journal of Musculoskelet Research, 6(3-4), 135-145.

[16] Waddell, G. and Burton, A.K. (2001) Occupational health guidelines for the management of low back pain at work: evidence review. Occupational Medicine, 51(2), 124135.

[17] Sjøgaard, G., Lundberg, U. and Kadefors, R. (2000) The role of muscle activity and mental load in the development of pain and degenerative processes at the muscle cell level during computer work. European Journal of Applied Physiology, 83(2-3), 99-105.

[18] Leyk, D. (2009) The preventive and therapeutic roles of regular physical activity. Deutsches Aerzteblat international, 106(44), 713-714.

[19] Baum, K., Selle, K., Leyk, D. and Essfeld, D. (1995) 
Comparison of blood pressure and heart rate responses to isometric exercise and passive muscle stretch in humans. European Journal of Applied Physiology, 70(3), 240-245.

[20] Davis, K.G. and Marras, W.S. (2000) The effects of motion on trunk biomechanics. Clinical Biomechanics, 15(10), 703-717.
[21] Finsen, L., Christensen, H. and Bakke, M. (1998) Musculoskeletal disorders among dentists and variation in dental work. Applied Ergonomics, 29(2), 119-125.

[22] Ratzon, N.Z., Yaros, T., Mizlik, A. and Kanner, T. (2000) Musculoskeletal symptoms among dentists in relation to work posture. Work, 15(4), 153-158. 